\title{
MANAGING CYBERSECURITY RESOURCES: A COST-BENEFIT ANALYSIS
}

\author{
A book by Lawrence A. Gordon and Martin Loeb
}

The book was just published by McGraw-Hill. A fundamental argument 1 throughout the book is that the proper use of economic concepts will allow organizations (in both the private and public sectors) to achieve a higher level of cybersecurity than otherwise possible. This argument is developed by providing an economic framework for:

- Determining the appropriate amount for an organization to invest in cybersecurity, and

- Procedures for efficiently allocating such resources to particular cybersecurity activities.

The book also provides chapters on The Business Case for Cybersecurity, Risk Management and Cybersecurity, Cybersecurity Auditing, and Cybersecurity's Role in National Security.

More information about the book can be found at:

www.rhsmith.umd.edu/faculty/lgordon/cybersecuritybook.htm.

Address for Correspondence:

Lawrence A. Gordon, Ph.D.

Ernst \& Young Alumni Professor of Managerial Accounting and Information Assurance

Director, Ph.D. Program

Affiliate Professor in University of Maryland Institute for Advanced Computer Studies

Robert H. Smith School of Business 
3359 Van Munching Hall

University of Maryland

College Park, MD 20742-1815

(301) 405-2255 TEL

(301) 314-9611 FAX

lgordon@rhsmith.umd.edu

http://www.rhsmith.umd.edu

http://www.rhsmith.umd.edu/faculty/lgordon/ 